\title{
WDR79 promotes the proliferation of non-small cell lung cancer cells via USP7-mediated regulation of the Mdm2-p53 pathway
}

\author{
Yang Sun ${ }^{1,5}$, Lanqin $\mathrm{Cao}^{2,5}$, Xunan Sheng ${ }^{1}$, Jieying Chen ${ }^{1}$, Yu Zhou ${ }^{1}$, Chao Yang ${ }^{1,3}$, Tanggang Deng ${ }^{1}$, Hongchang Ma ${ }^{1}$, Peifu Feng ${ }^{1}$,
} Jing Liü, Weihong Tan ${ }^{*, 1}$ and Mao Ye ${ }^{*, 1}$

WD repeat protein 79 (WDR79) is a member of the WD-repeat protein family and functions as a scaffold protein during telomerase assembly, Cajal body formation and DNA double strand break repair. We have previously shown that WDR79 is frequently overexpressed in cell lines and tissues derived from non-small cell lung cancer (NSCLC) and it accelerates cell proliferation in NSCLC. However, the detailed mechanism underlying the role of WDR79 in the proliferation of NSCLC cells remains unclear. Here, we report the discovery of a molecular interaction between WDR79 and USP7 and show its functional significance in linking the Mdm2-p53 pathway to the proliferation of NSCLC cells. We found that WDR79 colocalized and interacted with USP7 in the nucleus of NSCLC cells. This event, in turn, reduced the ubiquitination of Mdm2 and p53, thereby increasing the stability and extending the half-life of the two proteins. We further found that the functional effects of WDR79 depended upon USP7, because the knockdown of USP7 resulted in their attenuation. Finally, we demonstrated that WDR79 promoted the proliferation of NSCLC cells via USP7. Taken together, our findings reveal a novel molecular function of WDR79 and may lead to broadly applicable and innovative therapeutic avenues for NSCLC.

Cell Death and Disease (2017) 8, e2743; doi:10.1038/cddis.2017.162; published online 13 April 2017

The WDR79 gene on chromosome 17p13 encodes both an antisense transcript (WRAP53a) for 553 stabilization $^{1}$ and a protein (WDR79, WRAP53, WRAP53 $\beta$ or TCAB1) with six individual WD-repeat domains via the use of alternative transcriptional start sites. The protein WDR79 functions as a scaffold protein that is involved in telomerase localization, telomere and Cajal body assembly, and DNA double strand break repair. $^{2-8}$ The dysfunction of WDR79 has been implicated in human diseases. Specifically, germline mutations in WDR79 that affect the WD-repeat domain result in congenital dyskeratosis, ${ }^{9}$ and WDR79 overexpression has been observed in rectal cancer, ${ }^{10}$ head and neck carcinomas, ${ }^{11}$ squamous cell carcinoma, ${ }^{12}$ breast cancer ${ }^{13}$ and ovarian cancer. ${ }^{14}$ In a previous study, we also found that WDR79 is frequently overexpressed in cell lines and tissues derived from non-small cell lung cancer (NSCLC) and accelerates NSCLC cell proliferation. ${ }^{15}$ However, the detailed mechanism underlying the effect of WDR79 on the proliferation of NSCLC cells remains unclear.

Ubiquitin-specific proteases (USPs) constitute the largest subfamily of deubiquitinases, which consist of more than 60 members. They function as cysteine proteases to remove ubiquitin from specific protein substrates and allow protein salvage from proteasomal degradation or regulate their subcellular location and activation. USP7, a member of the
USP family, is first defined as Herpes virus associated cellular factor (HAUSP) and is critical for genome stability, epigenetic regulation, cell cycle, apoptosis, viral infection immunity and stem cell maintenance. ${ }^{16-20}$ Clinically, USP7 is reportedly associated with tumorigenesis, including prostate cancer, multiple myeloma cancer, ovarian cancer, breast cancer and NSCLC. ${ }^{20-24}$

As a tumour suppressor protein, p53 function is abnormal in over $50 \%$ of human cancers. ${ }^{25,26}$ In normal unstressed cells, p53 is a very unstable protein with a short half-life and maintained at a low level due to its rapid degradation via the ubiquitin-dependent proteasome pathway. ${ }^{27,28}$ However, in response to diverse cellular stress, such as DNA damage, hypoxia, telomeres shortening and oncogene activation, p53 is rapidly stabilized because its degradation is blocked, which results in cell cycle arrest, apoptosis and cellular senescence. ${ }^{29} \mathrm{Mdm} 2$ is the E3 ubiquitin ligase of p53 and binds to p53 to promote its degradation. ${ }^{30}$ Recent studies have revealed that USP7 is involved in the regulation of the p53-Mdm2 pathway. USP7 can bind Mdm2 or p53 via its $\mathrm{N}$-terminal and $\mathrm{C}$-terminal regions in a mutually exclusive manner, which consequently stabilizes the two proteins by removing ubiquitin. The in vitro binding affinity of USP7 for Mdm2 is several-fold higher than for p53, which implies that Mdm2 is the preferred substrate of USP7 in normal cellular homeostasis. In addition, USP7 may deubiquitinate p53 via

\footnotetext{
${ }^{1}$ Molecular Science and Biomedicine Laboratory, State Key Laboratory for Chemo/Biosensing and Chemometrics, College of Biology, College of Chemistry and Chemical Engineering, Collaborative Innovation Center for Molecular Engineering for Theranostics, Hunan University, Changsha, Hunan 410082 , China; ${ }^{2}$ Department of Gynecology, Xiangya Hospital, Central South University, Changsha, Hunan 410078, China; ${ }^{3}$ College of Life and Environmental Sciences, Gannan Normal University, Ganzhou, Jiangxi 341000, China and ${ }^{4}$ School of Life Sciences, State Key Laboratory of Medical Genetics, Central South University, Changsha, Hunan 410078, China

*Corresponding author: W Tan or M Ye, College of Biology, Hunan University, 1 Denggao Road, Yuelu District, Changsha, Hunan 410082 , China. Tel: +86 731 88821834 ; Fax: +86 731888 21894; E-mail: tan@chem.ufl.edu or yemaocsu@ hotmail.com

${ }^{5}$ These authors contributed equally to this work.

Received 03.12.16; revised 02.3.17; accepted 13.3.17; Edited by Z-X Xiao.
} 
Mdm2. ${ }^{31}$ However, upon DNA damage, ataxia telangiectasia mutated-dependent phosphorylation of Mdm2 decreased the binding affinity between USP7 and Mdm2, tilting the balance towards p53 stabilization. ${ }^{31-34}$

In this study, we report a new molecular process in which WDR79 interacts with USP7 to modulate the stability of Mdm2 and p53, which promotes the proliferation of NSCLC cells. In particular, we found that WDR79 colocalized and interacted with USP7 in the nucleus of NSCLC cells. This event, in turn, increased the stability and prolonged the half-life of Mdm2 and p53 by decreasing ubiquitination. We further found that the functional effects of WDR79 depended on USP7 and manifest a net outcome as promoting the proliferation of NSCLC cells. Taken together, our findings reveal a novel role of WDR79 in the proliferation of NSCLC cells and could pinpoint a new mechanism by which WDR79 and USP7 functionally interact to modulate the Mdm2-p53 pathway.

\section{Results}

WDR79 interacts with USP7. Our previous studies have shown that WDR79 is mainly located in the nuclei in NSCLC tissues and cell lines. Because of the limited knowledge regarding WDR79 function, we first characterized the functional domains of WDR79 by ELM (Eukaryotic Linear Motif) to get a clue on WDR79 function in nuclei. Four putative USP7 binding domains were identified in WDR79, one was located at the $\mathrm{N}$ terminus, and three at the $\mathrm{C}$ terminus (Figure 1a). To test the hypothesis that WDR79 is associated with USP7, we first examined the subcellular localization of WDR79 and USP7 by indirect immunofluorescence. Endogenous WDR79 and USP7 were labelled with their corresponding antibodies conjugated to DyLight 594 and 488, respectively. An intense yellow colour was observed in merged images of USP7 with WDR79 expression (Figure 1b), indicating that WDR79 and USP7 colocalized in the nucleus. To confirm that WDR79 indeed interacts with USP7 in vivo, Flag-WDR79 plasmid was

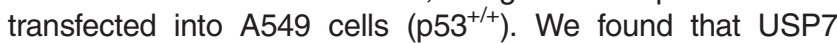
was co-immunoprecipitated by anti-Flag antibody in WDR79overexpressing cells but not in negative control cells transfected with the same amount of empty vector (Figure 1c). Next, the endogenous interaction between USP7 and WDR79 was also investigated by co-immunoprecipitation. As shown in Figures 1d and e, USP7 was detected in the anti-WDR79 immunoprecipitates from $\mathrm{H} 1299$ cells $\left(\mathrm{p} 53^{-/-}\right.$) and vice versa, but not in an isotype-matched negative control IgG. Collectively, these results indicate that WDR79 interacts with USP7 and that their interaction is independent of p53 expression.

WDR79 influences the protein level of Mdm2 and p53 but not their localization. USP7 is a key deubiquitinase involved in the regulation of the p53-Mdm2 pathway and its forced expression results in $\mathrm{p} 53$ and Mdm2 stabilization. ${ }^{18,34-37}$ Given the interaction between WDR79 and USP7 described above, we investigated the effect of WDR79 on the protein levels of Mdm2, p53 and USP7. To this end, we first overexpressed vector control and Flag-WDR79 in A549 cells and examined protein expression by western blotting. The results show that WDR79 overexpression significantly increases the protein levels of Mdm2 and p53 compared with the control (Figure 2a). Notably, the expression of Mdm2 is well known to be primarily controlled by $p 53 .{ }^{38,39}$ To exclude the effect of p53 on Mdm2 expression, we also performed the assay in p53-null H1299 cells and found that the protein levels of Mdm2 were still increased after Flag-WDR79 overexpression (Figure 2b), suggesting that the upregulation of Mdm2 by WDR79 is independent of p53 expression. Next, we measured the expression levels of Mdm2 and/or p53 in both A549 and H1299 cells whose endogenous WDR79 were knocked down using WDR79-specific short hairpin RNA (shRNA). As shown in Figures $2 \mathrm{c}$ and $\mathrm{d}$, knockdown of WDR79 led to a decrease in Mdm2 and/r p53 levels. However, changes in WDR79 expression did not appear to affect USP7 levels. To determine whether the regulation of Mdm2 and p53 by WDR79 is mediated at the level of gene transcription, we examined the mRNA levels of Mdm2 and p53 by real-time PCR after downregulation or overexpression of WDR79. We found that the levels of Mdm2 and p53 mRNA were not significantly affected by WDR79 (Figures $2 \mathrm{e}-\mathrm{h}$ ), indicating that the effect of WDR79 on the Mdm2 and p53 levels is not due to changes in their transcription, but more likely occurs at the posttranslational level (protein level). Furthermore, the protein levels and cellular localization of Mdm2, p53 and USP7 were further detected by immunofluorescent staining after WDR79 was knocked down in A549 and H1299 cells. As shown in Figure 3, the knockdown of WDR79 resulted in a weak fluorescent staining of Mdm2 and p53, but not USP7, which is consistent with the result that WDR79 downregulation significantly diminished endogenous levels of Mdm2 and p53. Meanwhile, the knockdown of WDR79 did not seem to change the cellular localization of Mdm2, p53 and USP7 compared to control A549 and H1299 cells (Figures 3a and b).

WDR79 stabilizes Mdm2 and p53 by protecting them from proteasome-mediated degradation. Approximately $80 \%$ of intracellular proteins are degraded by the ubiquitinproteasome system (UPS). To elucidate the mechanism by which WDR79 regulates the protein levels of Mdm2 and p53, A549 and H1299 cells were treated with the proteasome inhibitor MG132. As expected, in the absence of MG132, changes in the Mdm2 and p53 protein levels were accompanied by WDR79 overexpression or downregulation in A549 cells. However, MG132 treatment stabilized and eventually attenuated the changes caused by WDR79 (Figures 4a and b). Similar results were also obtained in H1299 cells (Figures $4 \mathrm{c}$ and $\mathrm{d}$ ), suggesting that WDR79 regulates Mdm2 and p53 levels in a proteasome-dependent manner.

Both Mdm2 and p53 have a short half-life and are strictly maintained at low levels by ubiquitin-mediated proteolytic degradation in unstressed cells. ${ }^{40,41}$ To assess the ability of WDR79 to regulate Mdm2 and p53 stability, A549 and H1299 cells with or without WDR79 knockdown were treated with cycloheximide to inhibit protein biosynthesis. Cell lysates collected at the indicated time points were analysed by western blotting, which revealed that the half-lives of Mdm2 and p53 were significantly shorter in WDR79-knockdown cells than in control cells (Figures $4 \mathrm{e}-\mathrm{h}$ ). The above results demonstrate that WDR79 stabilizes Mdm2 and p53 by preventing their proteasomal degradation. 


\section{a}

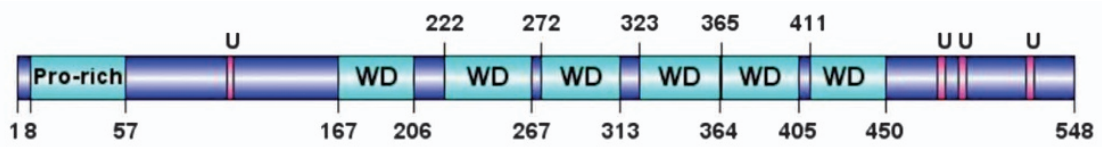

b

WDR79
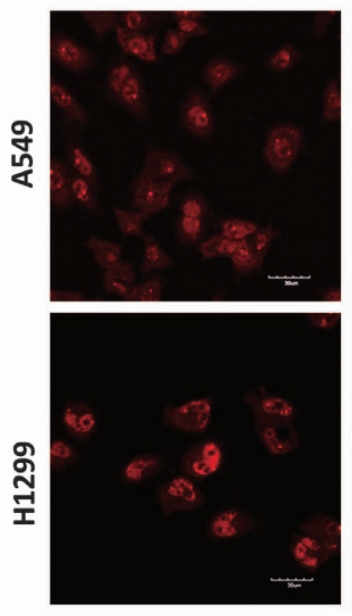

C

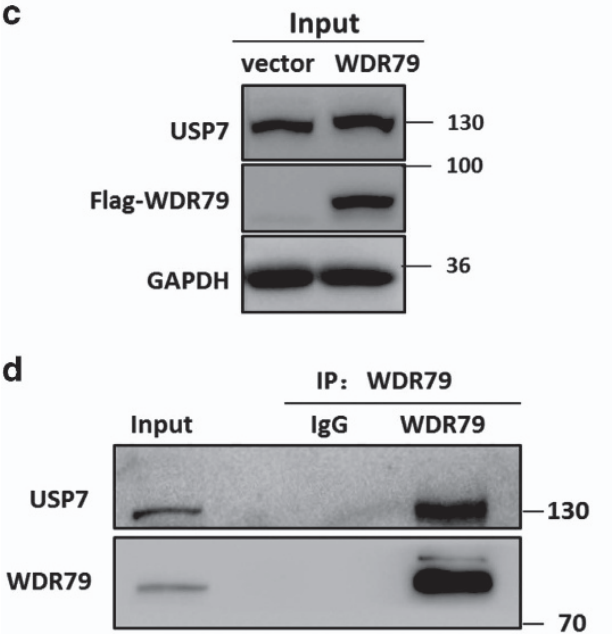

d

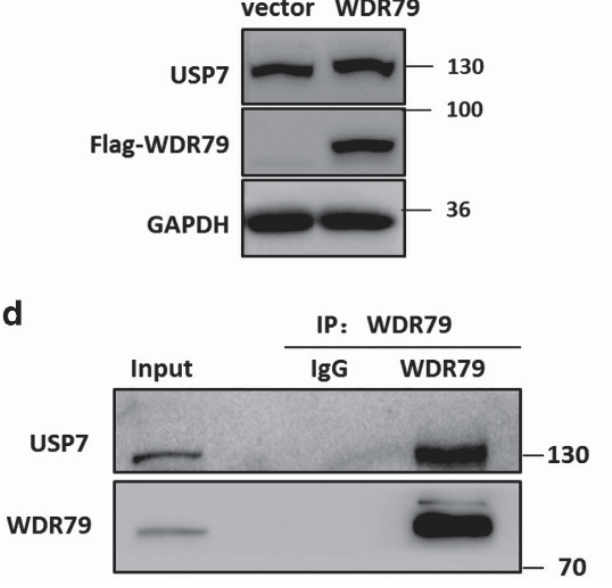

USP7
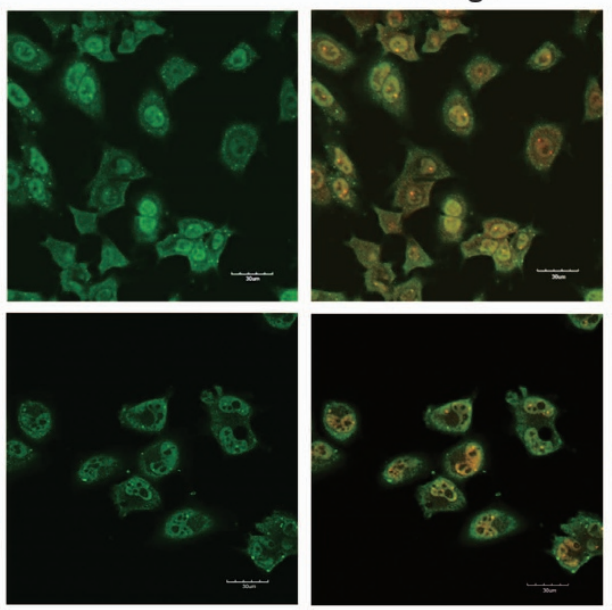

DAPI
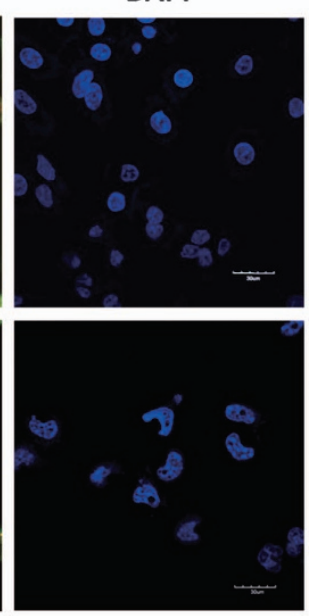

e
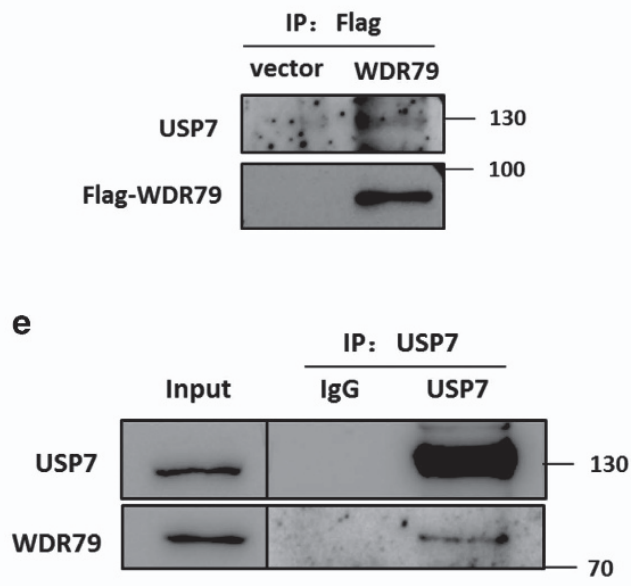

Figure 1 WDR79 interacts with USP7. (a) The predicted functional domains of WDR79 are shown (U: USP7 binding site). (b) A549 and H1299 cells were fixed and incubated with anti-WDR79 and anti-USP7 antibodies, followed by staining with DyLight 594 or Dylight 488-conjugated lgG. DAPI was used for nuclei staining (bars, $30 \mu \mathrm{m})$. (c-e) A549 cells transfected with the indicated constructs or H1299 cells were lysed and lysates were subject to immunoprecipitation with anti-Flag (c), anti-WDR79 (d), or anti-USP7 (e) antibodies. The immunoprecipitates were then blotted with the indicated antibodies

WDR79 is involved in the regulation of the ubiquitination of Mdm2 and p53. Mdm2 is a key negative regulator of p53 via its E3 ligase activity and itself subject to autoubiquitination. ${ }^{42,43}$ Because WDR79 influences the half-lives of Mdm2 and p53, we hypothesized that WDR79 may be involved in the regulation of their ubiquitination. To test this hypothesis, cells were treated with the proteasome inhibitor MG132 such that polyubiquitinated Mdm2 and p53 proteins were accumulated for detection. As shown in Figures $5 a-d$, Mdm2 and p53 ubiquitination was significantly increased when WDR79 was knocked down, whereas the overexpression of WDR79 decreased their ubiquitination in A549 cells. Similar results were obtained in $\mathrm{H} 1299$ cells (Figures $5 \mathrm{e}$ and f). Taken together, these findings suggest that WDR79 controls the stability of Mdm2 and p53 by modulating their ubiquitination prior to proteasomal degradation.

WDR79 mediates Mdm2 and p53 stabilization in a UPS7-dependent manner. WDR79 is a scaffold protein not known to possess any enzymatic activity. Because it interacts with and exerts effects similar to that of USP7 on Mdm2 and p53, we surmised that WDR79 acts via the deubiquitinating activity of USP7. To test this hypothesis, USP7 was knocked down with shRNA. As shown in Figures $6 a$ and $b$, knockdown of USP7 reduced the levels of Mdm2 and p53, a result consistent with that of a previous study. Moreover, the data revealed that knockdown of USP7 attenuated the stabilizing effect of WDR79 on Mdm2 and p53. 
a
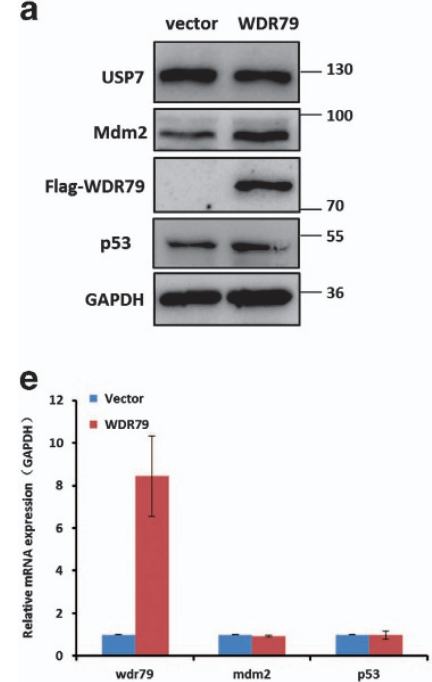

b

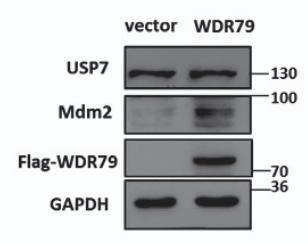

f

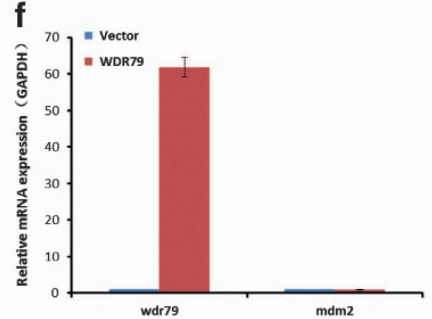

c

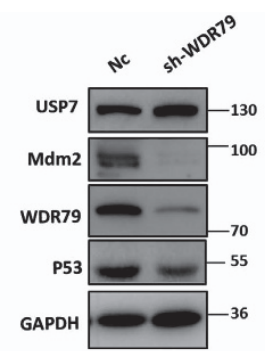

d

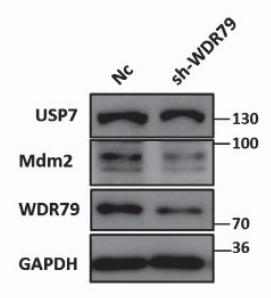

Figure 2 WDR79 influences the protein levels of Mdm2 and p53. (a and b) A549 (a) and H1299 (b) cells transfected with the indicated constructs for 24 h. Proteins in lysates were detected by western blotting using the indicated antibodies. (c and d) A549 (c) and H1299 (d) cells infected with lentivirus encoding the indicated shRNA were lysed and lysates were blotted with the indicated antibodies. (e and f) A549 (e) and H1299 (f) cells were transfected with the indicated constructs. Twenty-four hours later, the indicated mRNA was extracted and subjected to real-time PCR. ( $g$ and $\mathbf{~ h ) ~ A 5 4 9 ~ ( g ) ~ a n d ~ H 1 2 9 9 ~ ( h ) ~ c e l l s ~ w e r e ~ i n f e c t e d ~ w i t h ~ l e n t i v i r u s ~ e n c o d i n g ~ t h e ~ i n d i c a t e d ~ s h R N A . ~ T w e n t y - f o u r ~ h o u r s ~ l a t e r , ~}$ the indicated mRNA was extracted and subjected to real-time PCR

a

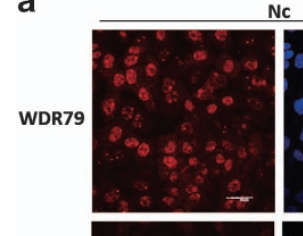

p53

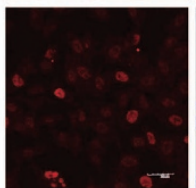

Mdm2

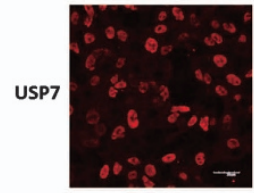

Dylight594
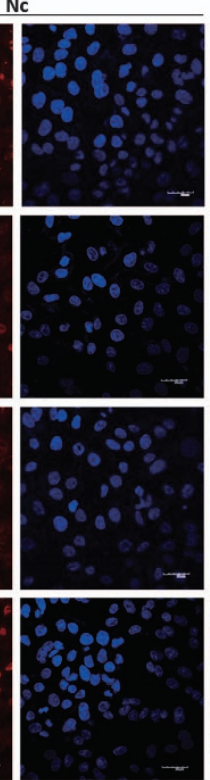

DAPI
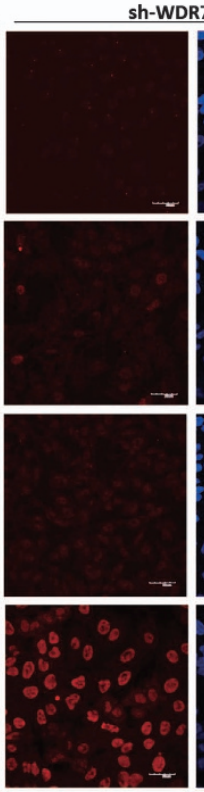

Dylight594 g

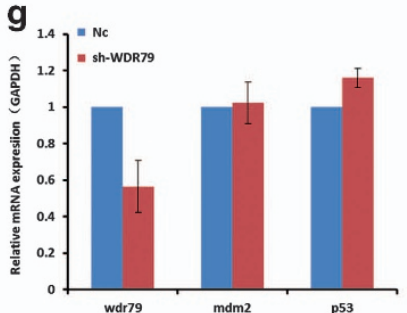

h

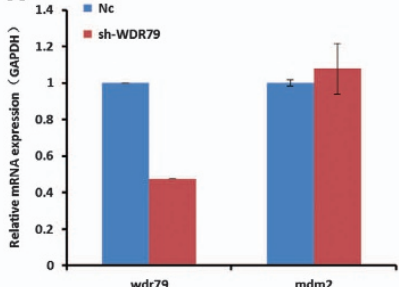




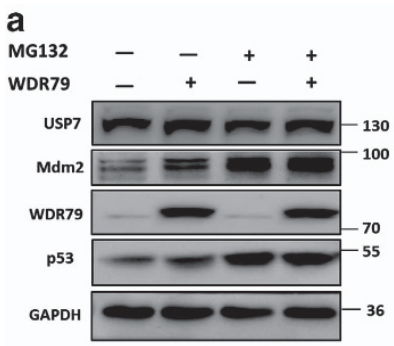

b

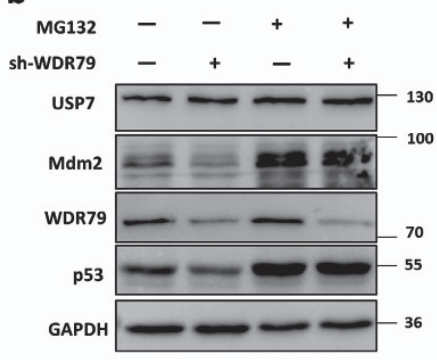

e
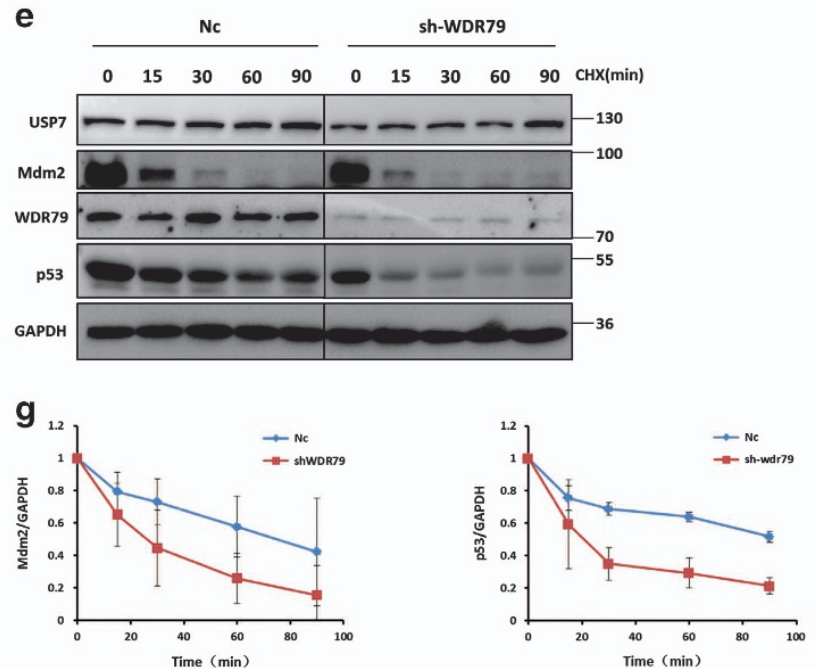

C

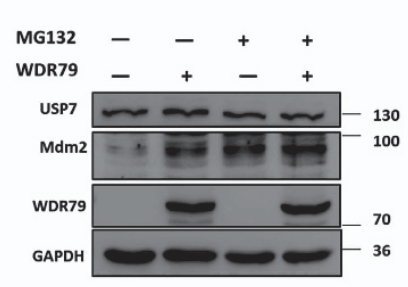

d

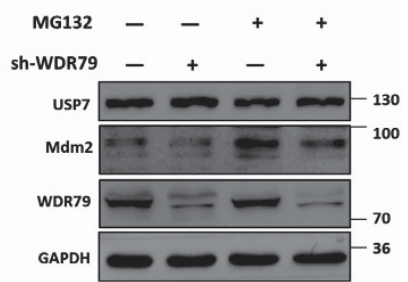

f
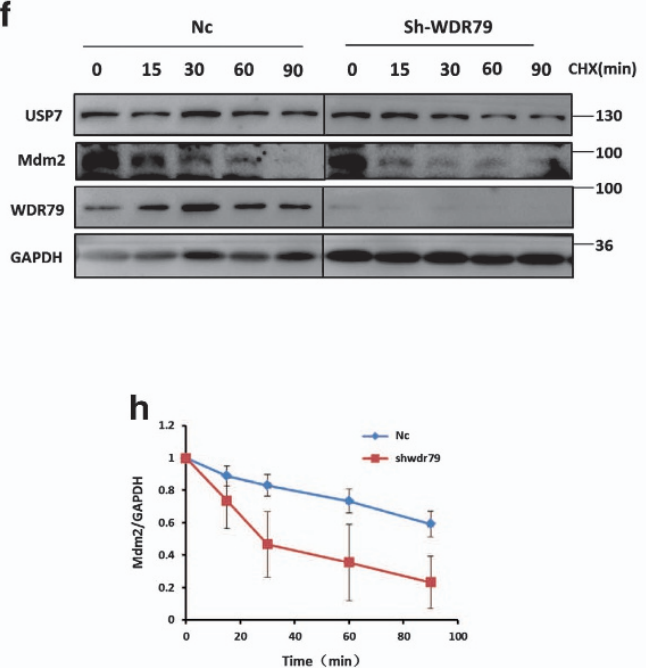

Figure 4 WDR79 stabilizes Mdm2 and p53 by protecting them from proteasome-mediated degradation. (a and $\mathbf{b})$ A549 cells transfected with the indicated constructs (a) or infected with lentivirus encoding the indicated shRNA (b) were treated with DMSO or MG132 for $6 \mathrm{~h}$. Expression of the indicated protein was examined by western blotting using the indicated antibodies. (c and d) H1299 cells transfected with the indicated constructs (c) or infected with lentivirus encoding the indicated shRNA (d) were treated with DMSO or MG132 for $6 \mathrm{~h}$. Expression of the indicated protein was examined by western blotting using the indicated antibodies. (e and f) A549 (e) and H1299 (f) cells infected with lentivirus encoding the indicated shRNA were treated with cycloheximide $(50 \mu \mathrm{g} / \mathrm{ml})$, harvested at the indicated point and then immunoblotted with the indicated antibodies. ( $\mathbf{g}$ and $\mathbf{h}$ ) Quantification of the p53 or Mdm2 protein levels relative to GAPDH. Intensities of blots were analysed by the ImageJ software

Because WDR79 affected the interaction of USP7 with Mdm2 and p53, we hypothesized that WDR79 is involved in USP7-mediated regulation of Mdm2 and p53. To test this hypothesis, Flag-USP7 was transfected into WDR79knockdown cells. As expected, the effect of USP7 on Mdm2 and p53 markedly decreased after WDR79 was knocked down (Figures 6e and f), suggesting that WDR79 plays an important role in USP7-mediated regulation of Mdm2 and p53. Overall, these data indicate that WDR79 and USP7 are close partners in the regulation of $\mathrm{Mdm} 2$ and $\mathrm{p53}$.

WDR79 promotes NSCLC cell proliferation through USP7. We have previously shown that WDR79 is frequently overexpressed in cell lines and tissues derived from NSCLC and it accelerates cell proliferation in NSCLC cell lines. To identify the necessity of USP7 for the growth-promoting effect of WDR79, we analysed cell growth using MTT assay. We found that WDR79 overexpression promoted cell proliferation compared with control cells, which is consistent with our previous results. However, USP7 downregulation effectively attenuated the growth-promoting effect of WDR79 (Figure 7a), indicating that WDR79-mediated cell growth depends on USP7.

\section{Discussion}

The WDR79 gene encodes two functional products via the use of alternative transcription start sites: one is an antisense transcript (WRAP53a) that stabilizes p53 by binding to the $5^{\prime}$ UTR of p53 mRNA, the other is a protein (WDR79, WRAP53, WRAP53 3 or TCAB1) with six individual WDrepeat domains that functions as a scaffold protein involved in the maintenance of Cajal bodies, telomere elongation and DNA repair. Previous studies have shown that WDR79 (WRAP53 $\beta$ ) protein cannot regulate p53 expression. ${ }^{44}$ Intriguingly, we found although WDR79 protein did not affect the transcription of p53 mRNA, it could stabilize p53 protein by interacting with deubiquitinating enzyme USP7 via the ubiquitin proteasome system in the A549 NSCLC cell line. We provide several lines of evidence to support this conclusion. First, the overexpression of full-length WDR79 protein without overlap sequences with p53 mRNA can increase p53 level. Second, neither the overexpression nor the knockdown of WDR79 influenced the transcription of p53 mRNA. Third, the proteasome inhibitor MG132 abolished the effect of WDR79 on p53, indicating that WDR79 regulates protein level of $p 53$. Fourth, WDR79 affected p53 protein stability in a half-life assay using the protein synthesis inhibitor 
a

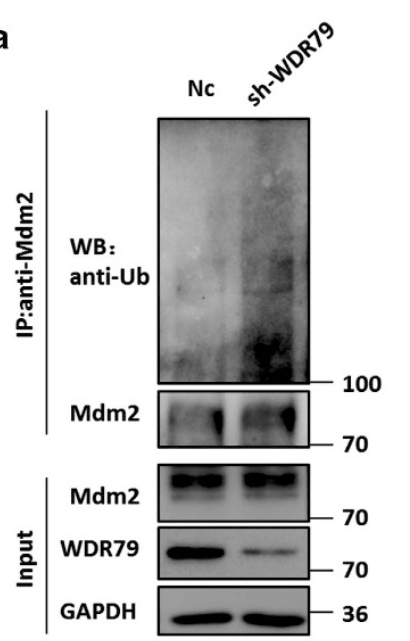

d

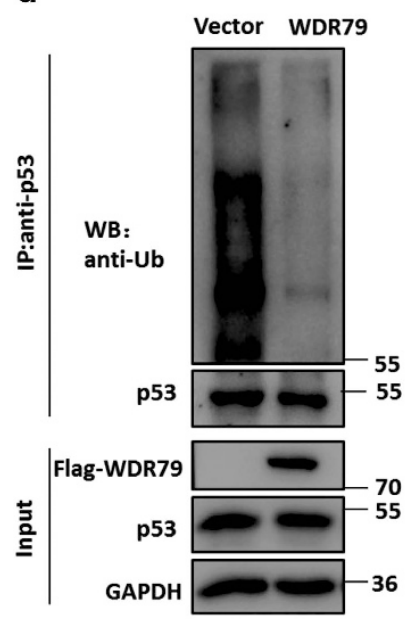

b
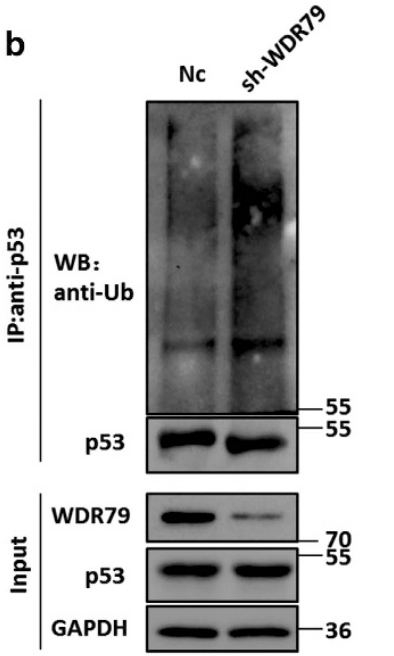

e

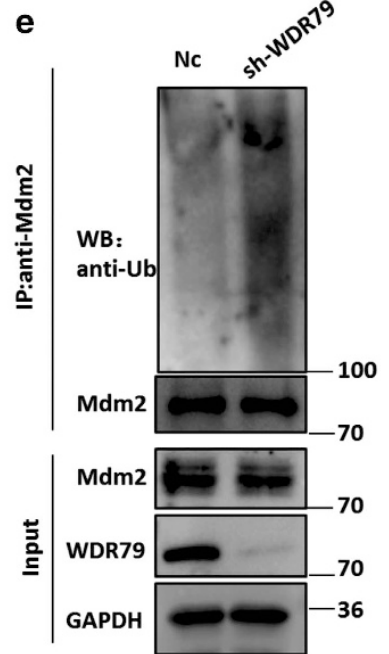

C

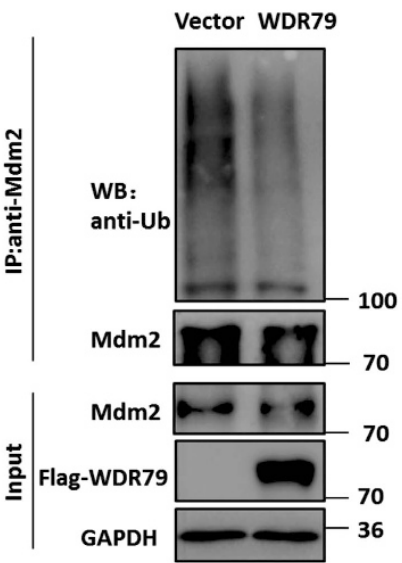

f

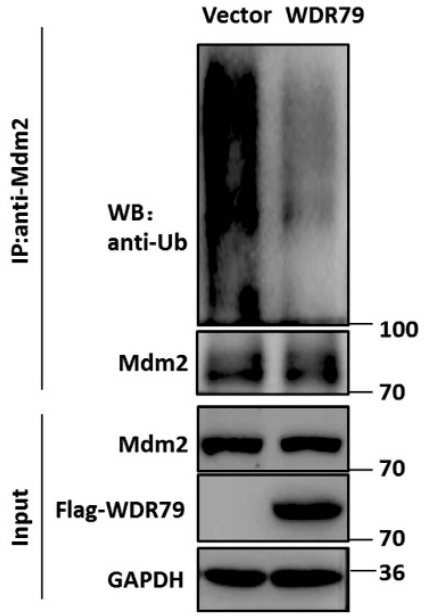

Figure 5 WDR79 affects the ubiquitination of Mdm2 and p53. (a and $\mathbf{b})$ A549 cells infected with lentivirus encoding the indicated shRNA were treated with MG132 for $6 \mathrm{~h}$. Lysates were immunoprecipitated with anti-Mdm2 (a) or anti-p53 (b) antibody. The ubiquitination of the Mdm2 and p53 was analysed by western blotting using anti-ubiquitin antibody. (c and d) A549 cells transfected with the indicated constructs were treated with MG132 for $6 \mathrm{~h}$. The ubiquitination of Mdm2 and p53 was analysed as above. (e and f) $\mathrm{H} 1299$ cells infected with lentivirus encoding the indicated shRNA (e) or transfected with the indicated constructs (f) were treated with MG132 for 6 h. The ubiquitination of Mdm2 and p53 was analysed as above

cycloheximide. Fifth, WDR79 interacts with USP7. Moreover, knockdown of USP7 attenuated the effect of WDR79 on p53, revealing that WDR79 acts as a scaffold protein to affect p53 via USP7.

The WD-repeat domain is defined by four or more repeating units that contain a conserved core of 40-60 amino acids that begins with a glycine-histidine dipeptide and ends with a tryptophan-aspartic acid (WD) dipeptide. ${ }^{45}$ The human genome encodes $\sim 300$ proteins containing WD-repeat domains that are thought to fold into $\beta$-propeller structures and form a platform without any catalytic activity for the assembly of multiple protein complexes. ${ }^{46}$ Many studies have revealed that WD-repeat proteins are involved in ubiquitinproteasome process. Especially, some F-box proteins were found to mediate ubiquitin-mediated proteolysis through WD-repeat domains, such as FBW8, FBXW2, beta-TrCP and F-box/WD repeat containing protein 7(FBW7) ${ }^{47-53}$ Moreover, some WD-repeat proteins were implicated in interaction with 20 different USPs in a proteomic screen. ${ }^{54}$ For example, WDR48 as well as two smaller homologues, USP12 and USP46, have recently been demonstrated to function as activators of USP1 ${ }^{55,56}$ Due to its six individual WD-repeat domains, WDR79 belongs to the WD-repeat protein family. Previous studies show that WDR79 orchestrates the ubiquitin response, which is critical for DNA double strand break repair, as a scaffold protein by facilitating the interaction between E3 ligase RNF8 and its partner. ${ }^{6}$ Here we discovered a novel molecular function of WDR79: it is involved in the regulation of Mdm2 and p53 deubiquitination by interacting with USP7. Specifically, the deubiquitinating activity of USP7 depends on WDR79. The knockdown of WDR79 not only abolished the effect of USP7 on Mdm2 and p53 but also decreased the binding of USP7 to Mdm2 and p53. From a functional perspective, we speculate that, WDR79, a scaffold protein with multiple WD-repeat domains, may form a platform to actively recruit or tether USP7 and its targets Mdm2 and/or 


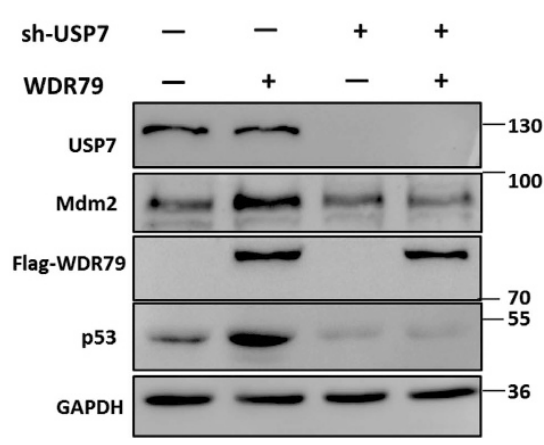

C
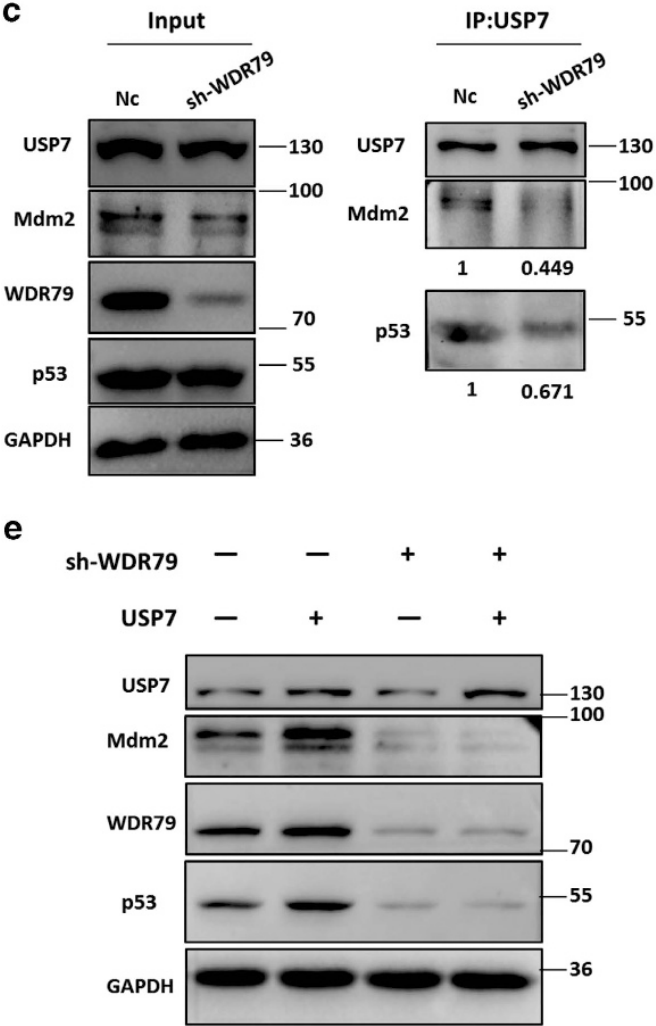

b

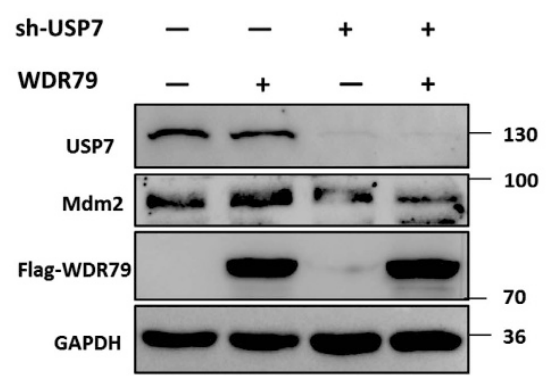

d

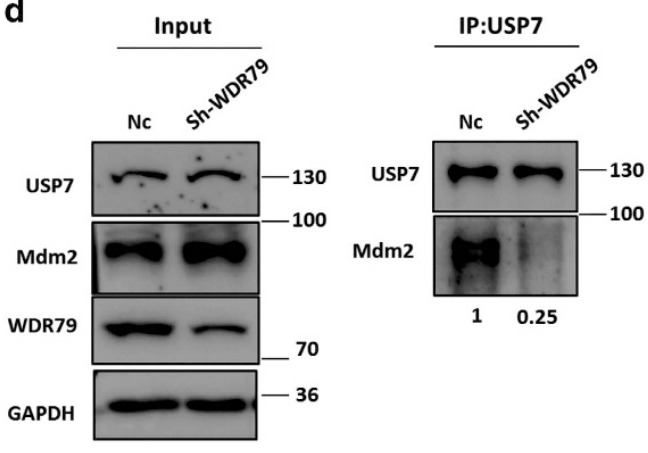

f

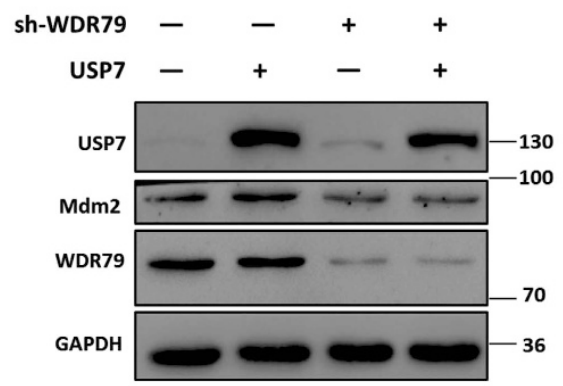

Figure 6 WDR79 mediates Mdm2 and p53 stabilization in a UPS7-dependent manner. (a and b) A549 (a) or H1299 (b) cells infected with lentivirus encoding the indicated shRNA were transfected with the indicated constructs. Lysates were blotted with the indicated antibodies. (c and d). A549 (c) or H1299 (d) cells infected with the lentivirus encoding the indicated shRNA were treated with MG132 for $6 \mathrm{~h}$. Immunoprecipitation were performed with anti-USP7 antibody. The immunoprecipitates were then blotted with the indicated antibodies. (e and f) A549 (e) or H1299 (f) cells infected with lentivirus encoding the indicated shRNA were transfected with the indicated constructs. Lysates were blotted with the indicated antibodies

p53 from the nucleoplasm, which facilitates the USP7mediated stabilization of Mdm2 and p53.

WDR79 mediates the stability of Mdm2 and p53 via USP7, but the latter two proteins execute opposing functions in various cellular settings. ${ }^{42,57}$ This dichotomy raises an interesting question: how does WDR79 promote the proliferation of NSCLC cells? Based on our results, we found that the effect of WDR79 on Mdm2 is far stronger than that on p53. Because USP7 mainly deubiquitinates and stabilizes Mdm2 in unstressed cells, we believe that WDR79 may stimulate the USP7-Mdm2 axis more effectively than the USP7-p53 counterpart, thereby facilitating the proliferation of NSCLC cells despite the functional antagonism between Mdm2 and p53.
In summary, we have identified WDR79 as an upstream factor of USP7 and uncovered a new pathway from WDR79USP7 interaction to growth promotion in NSCLC cells. Based on our results, we propose a model that accounts for the function of WDR79 via USP7 regulation. WDR79 may form a platform to recruit or tether USP7 and its targets Mdm2 and/or p53 from the nucleoplasm, which potentiates USP7 ability to deubiquitinate Mdm2 and p53, thereby stabilizing both Mdm2 and p53 and extending their half-lives. Given the opposite functions between p53 and Mdm2, we reason that the WDR79 - USP7 interaction may render USP7 more active towards Mdm2 than p53, a dynamic interplay that ultimately results in the proliferation of NSCLC cells (Figure 7b). This 


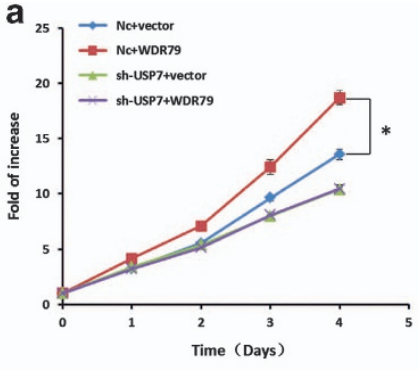

b

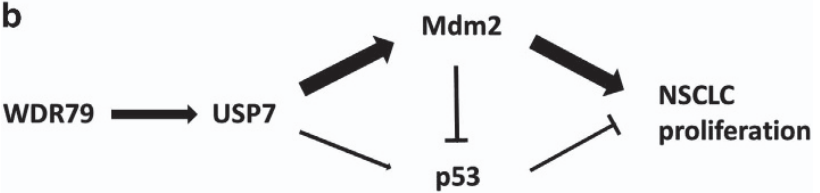

Figure 7 WDR79 promotes NSCLC cell proliferation via USP7. (a) A549 cells infected with lentivirus encoding the indicated shRNA were transfected with the indicated constructs. Cell proliferation was analysed with MTT at the indicated time points. $\left(n=3\right.$; $\left.{ }^{*} P<0.05\right)$. (b) A schematic illustration of the working model that WDR79 promotes the proliferation of NSCLC cells via USP7-mediated regulation of the Mdm2-p53 pathway

view reinforces the importance of the relative effects on the Mdm2-p53 pathway in terms of their antagonism ${ }^{43}$ and links the complexity of additional factors to this central and competitive regulation. In conclusion, the role of the WDR79 USP7 interaction is a novel finding. Further investigations of this molecular process and the elucidation of its precise functions in the context of protein complex assembly and specific signal transmissions should yield novel insights into the activities of cancer-associated pathway.

\section{Materials and Methods \\ Cell culture and reagents. Human NSCLC cell lines (H1299 and A549 cells) were cultured in RPMI-1640 medium (Gibco BRL Co. Ltd., Grand Island, NY, USA) with $10 \%$ fetal bovine serum (Gibco BRL Co. Ltd.) at $37^{\circ} \mathrm{C}$ in $5 \% \mathrm{CO}_{2}$ humidified incubators. Antibodies against p53 (DO-1), and Mdm2 (SMP14) were from Santa Cruz Biotechnology (CA, USA). The Mdm2 antibody (Ab-4) was from Millipore (Darmstadt, Germany), the USP7 and WDR79 antibodies were from Bethyl Laboratories (Montgomery, TX, USA), the GAPDH antibody was from KangChen Bio-tech Inc (Shanghai, China), and the antibody against ubiquitin (6C1.17) was from BD Pharmingen (Franklin Lakes, NJ, USA).}

Plasmids and transfection. To overexpress WDR79 or USP7 the full-length mRNA sequences were PCR-amplified from human cDNA and subcloned into pCMV-Tag2B to create Flag-tagged WDR79 or USP7 expression plasmid.

To stably knock down endogenous WDR79 in some cases, we used lentiviruspackaging shRNA expression vector (purchased from Gene Pharma, Shanghai, China) to infect cells. WDR79 shRNA target sequences were $5^{\prime}$-AATCAGCGCATCT ACTTCGAT-3'. The control shRNA sequence was 5'-TTCTCCGAACGTGTCAC GTTTC-3'.

Immunoprecipitation assay. Immunoprecipitations were performed with Universal Magnetic Co-IP Kit (Active Motif, Carlsbad, CA, USA). First, $1 \mathrm{mg}$ of crude extract was incubated with $3 \mu \mathrm{g}$ of a relevant primary antibody or an isotypematched negative control IgG overnight at $4{ }^{\circ} \mathrm{C}$. Second, samples were incubated with $30 \mu$ of magnetic beads conjugated with protein G19 for $1 \mathrm{~h}$ room temperature and washed three times with Co-IP/wash buffer. Next, precipitated proteins were dissolved in $30 \mu \mathrm{l} 2 \times$ SDS protein loading buffer, boiled for $10 \mathrm{~min}$ at $95^{\circ} \mathrm{C}$, and subjected to western blot analysis.

Western blot assay. Following the aforementioned cellular treatments, cells were collected and lysed with M-PER buffer containing protease inhibitors (Pierce,
Rock ford, IL, USA). Samples were resolved on $8-10 \%$ SDS-PAGE gels and transferred to nitrocellulose-membranes and blocked with $5 \%$ non-fat milk. Primary antibodies were incubated with the membranes overnight at $4{ }^{\circ} \mathrm{C}$. Subsequently, membranes were washed and incubated with appropriate horse-radish peroxidaseconjugated secondary antibodies (Santa Cruz, CA, USA). Blots were developed with Chemiluminescence Detection Kit (Pierce).

Indirect immunofluorescence assay. A549 and $\mathrm{H} 1299$ cells were fixed with $4 \%$ paraformaldehyde for $30 \mathrm{~min}$, permeabilized with $0.2 \%$ Triton X-100 for $15 \mathrm{~min}$, blocked with $5 \%$ bovine serum albumin, and then incubated with anti-WDR79 (Bethyl Laboratories, Inc., Montgomery, TX, USA), anti-p53, anti-MDM2 (Santa Cruz) or anti-USP7 (Bethyl Laboratories, Inc.) antibodies at $4{ }^{\circ} \mathrm{C}$ overnight, followed by a DyLight 594-conjugated or DyLight 488-conjugated secondary antibody (ImmunoReagents, Inc., Raleigh, NC, USA). Cells were stained with 2-(4Amidinophenyl)-6-indolecarbamidinedihydrochloride (DAPI) (Beyotime Biotechnology, Haimen, China) for $10 \mathrm{~min}$, and the images were acquired with a confocalmicroscope.

Protein half-life assay. Cells were treated with cycloheximide $(50 \mu \mathrm{g} / \mathrm{ml})$ for various periods to block protein synthesis. Crude extracts were prepared and protein levels were assessed by WB analysis.

In vivo ubiquitination assay. Cells pretreated with $25 \mu \mathrm{M} M \mathrm{MG} 132$ for $6 \mathrm{~h}$ were lysed and incubated with anti-Mdm2 or anti-p53 antibodies overnight at $4{ }^{\circ} \mathrm{C}$. Then $30 \mu$ beads conjugated with protein $\mathrm{G}$ were added and incubated for $1 \mathrm{~h}$ at room temperature. The immunoprecipitates were dissolved in $30 \mu \mathrm{l} 2 \times$ SDS protein loading buffer and analysed by WB using an anti-ubiquitin antibody.

Cell proliferation assay. Cells were seed in 96-well culture plates, 3-(4, 5dimethylthiazol-2-yl)-2, 5-diphenyltetrazolium bromide (Sigma, St. Louis, MO, USA) solution was added to each well at indicated time point and incubated at $37^{\circ} \mathrm{C}$ for another $4 \mathrm{~h}$. Supernatants were then aspirated, and the formazan product was dissolved with $100 \mu \mathrm{l}$ dimethyl sulfoxide. The absorbance was measured at a wavelength of $570 \mathrm{~nm}$ with a microplate reader (Bio-Tek, Doraville, GA, USA).

\section{Conflict of Interest}

The authors declare no conflict of interest.

Acknowledgements. This work was supported by grants from the National Basic Research Program of China (no. 2013CB932702), the National Natural Science Foundation of China (81171950, 81272220, 81402304 and 81672760), the Interdisciplinary Research Program of Hunan University and the Program for New Century Excellent Talents in University (NCET-13-0195).

1. Garcia-Closas M, Kristensen V, Langerod A, Qi Y, Yeager M, Burdett $L$ et al. Common genetic variation in TP53 and its flanking genes, WDR79 and ATP1B2, and susceptibility to breast cancer. Int $J$ Cancer 2007; 121: 2532-2538.

2. Tycowski KT, Shu MD, Kukoyi A, Steitz JA. A conserved WD40 protein binds the Cajal body localization signal of scaRNP particles. Mol Cell 2009; 34: 47-57.

3. Batista LF, Pech MF, Zhong FL, Nguyen HN, Xie KT, Zaug AJ et al. Telomere shortening and loss of self-renewal in dyskeratosis congenita induced pluripotent stem cells. Nature 2011; 474: 399-402.

4. Stern JL, Zyner KG, Pickett HA, Cohen SB, Bryan TM. Telomerase recruitment requires both TCAB1 and Cajal bodies independently. Mol Cell Biol 2012; 32: 2384-2395.

5. Vogan JM, Collins K. Dynamics of human telomerase holoenzyme assembly and subunit exchange across the cell cycle. J Biol Chem 2015; 290: 21320-21335.

6. Henriksson S, Rassoolzadeh H, Hedstrom E, Coucoravas C, Julner A, Goldstein M et al. The scaffold protein WRAP53beta orchestrates the ubiquitin response critical for DNA double-strand break repair. Genes Dev 2014; 28: 2726-2738.

7. Venteicher AS, Abreu EB, Meng Z, McCann KE, Terns RM, Veenstra TD et al. A human telomerase holoenzyme protein required for Cajal body localization and telomere synthesis. Science 2009; 323: 644-648.

8. Venteicher AS, Artandi SE. TCAB1 driving telomerase to Cajal bodies. Cell Cycle 2009; 8 : 1329-1331.

9. Zhong F, Savage SA, Shkreli M, Giri N, Jessop L, Myers T et al. Disruption of telomerase trafficking by TCAB1 mutation causes dyskeratosis congenita. Genes Dev 2011; 25: 11-16.

10. Zhang H, Wang DW, Adell G, Sun XF. WRAP53 is an independent prognostic factor in rectal cancer-a study of Swedish clinical trial of preoperative radiotherapy in rectal cancer patients. BMC Cancer 2012; 12: 294. 
11. Sun CK, Luo XB, Gou YP, Hu L, Wang K, Li C et al. TCAB1: a potential target for diagnosis and therapy of head and neck carcinomas. Mol Cancer 2014; 13: 180.

12. Rao X, Huang D, Sui X, Liu G, Song X, Xie J et al. Overexpression of WRAP53 is associated with development and progression of esophageal squamous cell carcinoma. PLOS ONE 2014; 9: e91670.

13. Silwal-Pandit L, Russnes H, Borgen E, Skarpeteig V, Moen Vollan HK, Schlichting E et al The sub-cellular localization of WRAP53 has prognostic impact in breast cancer. PLOS ONE 2015; 10: e0139965.

14. Hedstrom E, Pederiva C, Farnebo J, Nodin B, Jirstrom K, Brennan DJ et al. Downregulation of the cancer susceptibility protein WRAP53beta in epithelial ovarian cancer leads to defective DNA repair and poor clinical outcome. Cell Death Dis 2015; 6: e1892.

15. Sun Y, Yang $C$, Chen J, Song X, Li Z, Duan M et al. Overexpression of WDR79 in non-small cell lung cancer is linked to tumour progression. J Cell Mol Med 2016; 20: 698-709.

16. Nicholson B, Suresh Kumar KG. The multifaceted roles of USP7: new therapeutic opportunities. Cell Biochem Biophys 2011; 60: 61-68.

17. Nijman SM, Luna-Vargas MP, Velds A, Brummelkamp TR, Dirac AM, Sixma TK et al. A genomic and functional inventory of deubiquitinating enzymes. Cell 2005; 123: 773-786.

18. Li M, Brooks CL, Kon N, Gu W. A dynamic role of HAUSP in the p53-Mdm2 pathway. Mol Cell 2004; 13: 879-886.

19. Marchenko ND, Wolff S, Erster S, Becker K, Moll UM. Monoubiquitylation promotes mitochondrial p53 translocation. EMBO J 2007; 26: 923-934.

20. Zhang C, Lu J, Zhang QW, Zhao W, Guo JH, Liu SL et al. USP7 promotes cell proliferation through the stabilization of Ki-67 protein in non-small cell lung cancer cells. Int $J$ Biochem Cell Biol 2016; 79: 209-221.

21. Song MS, Salmena L, Carracedo A, Egia A, Lo-Coco F, Teruya-Feldstein J et al. The deubiquitinylation and localization of PTEN are regulated by a HAUSP-PML network. Nature 2008; 455: 813-817.

22. Chauhan D, Tian Z, Nicholson B, Kumar KG, Zhou B, Carrasco R et al. A small molecule inhibitor of ubiquitin-specific protease-7 induces apoptosis in multiple myeloma cells and overcomes bortezomib resistance. Cancer Cell 2012; 22: 345-358.

23. Qin D, Wang W, Lei H, Luo H, Cai H, Tang C et al. CDDO-Me reveals USP7 as a novel target in ovarian cancer cells. Oncotarget 2016; 7: 77096-77109.

24. Wang Q, Ma S, Song N, Li X, Liu L, Yang S et al. Stabilization of histone demethylase PHF8 by USP7 promotes breast carcinogenesis. J Clin Invest 2016; 126: 2205-2220.

25. Hollstein M, Rice K, Greenblatt MS, Soussi T, Fuchs R, Sorlie T et al. Database of p53 gene somatic mutations in human tumors and cell lines. Nucleic Acids Res 1994; 22: 3551-3555.

26. Vaughan C, Pearsall I, Yeudall A, Deb SP, Deb S. p53: its mutations and their impact on transcription. Subcell Biochem 2014; 85: 71-90.

27. Hock AK, Vousden KH. The role of ubiquitin modification in the regulation of p53. Biochim Biophys Acta 2014; 1843: 137-149.

28. Brooks CL, Gu W. p53 regulation by ubiquitin. FEBS Lett 2011; 585: 2803-2809.

29. Pflaum J, Schlosser S, Muller M. p53 Family and Cellular Stress Responses in Cancer. Front Oncol 2014; 4: 285.

30. Fang S, Jensen JP, Ludwig RL, Vousden KH, Weissman AM. Mdm2 is a RING fingerdependent ubiquitin protein ligase for itself and p53. J Biol Chem 2000; 275: 8945-8951.

31. Brooks CL, Li M, Hu M, Shi Y, Gu W. The p53 - Mdm2 - HAUSP complex is involved in p53 stabilization by HAUSP. Oncogene 2007; 26: 7262-7266.

32. Kon N, Kobayashi Y, Li M, Brooks CL, Ludwig T, Gu W. Inactivation of HAUSP in vivo modulates p53 function. Oncogene 2010; 29: 1270-1279.

33. Nicholson B, Marblestone JG, Butt TR, Mattern MR. Deubiquitinating enzymes as novel anticancer targets. Future Oncol 2007; 3: 191-199.

34. Sheng Y, Saridakis V, Sarkari F, Duan S, Wu T, Arrowsmith CH et al. Molecular recognition of p53 and MDM2 by USP7/HAUSP. Nat Struct Mol Biol 2006; 13: 285-291.

35. Li M, Chen D, Shiloh A, Luo J, Nikolaev AY, Qin J et al. Deubiquitination of p53 by HAUSP is an important pathway for p53 stabilization. Nature 2002; 416: 648-653.

36. Hu M, Gu L, Li M, Jeffrey PD, Gu W, Shi Y. Structural basis of competitive recognition of p53 and MDM2 by HAUSP/USP7: implications for the regulation of the p53-MDM2 pathway. PLOS Biol 2006; 4: e27.

37. Ye M, Tang Y, Tang S, Liu J, Wu K, Yao S et al. STIP is a critical nuclear scaffolding protein linking USP7 to p53-Mdm2 pathway regulation. Oncotarget 2015; 6: 34718-34731.

38. Barak Y, Juven T, Haffner R, Oren M. mdm2 expression is induced by wild type p53 activity. EMBO J 1993; 12: 461-468.
39. Picksley SM, Lane DP. The p53-mdm2 autoregulatory feedback loop: a paradigm for the regulation of growth control by p53? Bioessays 1993; 15: 689-690.

40. Olson DC, Marechal V, Momand J, Chen J, Romocki C, Levine AJ. Identification and characterization of multiple mdm-2 proteins and mdm-2-p53 protein complexes. Oncogene 1993; 8: 2353-2360.

41. Wen W, Peng C, Kim MO, Ho Jeong C, Zhu F, Yao K et al. Knockdown of RNF2 induces apoptosis by regulating MDM2 and p53 stability. Oncogene 2014; 33: 421-428.

42. Alarcon-Vargas D, Ronai Z. p53-Mdm2-the affair that never ends. Carcinogenesis 2002; 23 : 541-547.

43. Brooks CL, Gu W. p53 ubiquitination: Mdm2 and beyond. Mol Cell 2006; 21: 307-315.

44. Mahmoudi S, Henriksson S, Corcoran M, Mendez-Vidal C, Wiman KG, Farnebo M. Wrap53, a natural p53 antisense transcript required for p53 induction upon DNA damage. Mol Cell 2009; 33: 462-471.

45. Li D, Roberts R. WD-repeat proteins: structure characteristics, biological function, and their involvement in human diseases. Cell Mol Life Sci 2001; 58: 2085-2097.

46. Smith TF, Gaitatzes C, Saxena K, Neer EJ. The WD repeat: a common architecture for diverse functions. Trends Biochem Sci 1999; 24: 181-185.

47. Wang H, Chen Y, Lin P, Li L, Zhou G, Liu G et al. The CUL7/F-box and WD repeat domain containing 8 (CUL7/Fbxw8) ubiquitin ligase promotes degradation of hematopoietic progenitor kinase 1. J Biol Chem 2014; 289: 4009-4017.

48. Wang CC, Lo HF, Lin SY, Chen H. RACK1 (receptor for activated C-kinase 1) interacts with FBW2 (F-box and WD-repeat domain-containing 2) to up-regulate GCM1 (glial cell missing 1) stability and placental cell migration and invasion. Biochem J 2013; 453: 201-208.

49. Margottin F, Bour SP, Durand H, Selig L, Benichou S, Richard V et al. A novel human WD protein, h-beta TrCp, that interacts with HIV-1 Vpu connects CD4 to the ER degradation pathway through an F-box motif. Mol Cell 1998; 1: 565-574.

50. Wu C, Ghosh S. beta-TrCP mediates the signal-induced ubiquitination of lkappaBbeta. J Bio Chem 1999; 274: 29591-29594.

51. Shirane M, Hatakeyama S, Hattori K, Nakayama K, Nakayama K. Common pathway for the ubiquitination of IkappaBalpha, IkappaBbeta, and IkappaBepsilon mediated by the F-box protein FWD1. J Biol Chem 1999; 274: 28169-28174.

52. Kitagawa M, Hatakeyama S, Shirane M, Matsumoto M, Ishida N, Hattori K et al. An F-box protein, FWD1, mediates ubiquitin-dependent proteolysis of beta-catenin. EMBO J 1999; 18 2401-2410.

53. Hatakeyama S, Kitagawa M, Nakayama K, Shirane M, Matsumoto M, Hattori K et al. Ubiquitin-dependent degradation of lkappaBalpha is mediated by a ubiquitin ligase Skp1/Cul 1/F-box protein FWD1. Proc Natl Acad Sci USA 1999; 96: 3859-3863.

54. Sowa ME, Bennett EJ, Gygi SP, Harper JW. Defining the human deubiquitinating enzyme interaction landscape. Cell 2009; 138: 389-403.

55. Cohn MA, Kowal P, Yang K, Haas W, Huang TT, Gygi SP et al. A UAF1-containing multisubunit protein complex regulates the Fanconi anemia pathway. Mol Cell 2007; 28: 786-797.

56. Cohn MA, Kee Y, Haas W, Gygi SP, D'Andrea AD. UAF1 is a subunit of multiple deubiquitinating enzyme complexes. J Biol Chem 2009; 284: 5343-5351.

57. Kruse JP, Gu W. Modes of p53 regulation. Cell 2009; 137: 609-622.

(i) Cell Death and Disease is an open-access journal published by Nature Publishing Group. This work is licensed under a Creative Commons Attribution 4.0 International License. The images or other third party material in this article are included in the article's Creative Commons license, unless indicated otherwise in the credit line; if the material is not included under the Creative Commons license, users will need to obtain permission from the license holder to reproduce the material. To view a copy of this license, visit http://creativecommons.org/licenses/by/4.0/

(C) The Author(s) 2017 